\title{
b-jet production via Reggeized gluon fusion at Tevatron and LHC
}

Vladimir Saleev *

Samara State University

E-mail: saleev@samsu.ru

\section{Alexandra Shipilova ${ }^{\dagger}$}

Samara State University

E-mail: alexshipilova@samsu.ru

\begin{abstract}
We study inclusive $b$-jet and $b \bar{b}$-dijet production at the LHC and Tevatron invoking the hypothesis of gluon Reggeization in $t$-channel exchanges at high energy. The $b$-jet cross section includes contributions from open $b$-quark production in quasi-multi-Regge kinematics and from $b$-quark production via gluon-to-bottom-pair fragmentation within multi-Regge kinematics. We find good agreement with data by the ATLAS and CMS Collaborations at the LHC at the hadronic c.m. energy of $\sqrt{S}=7 \mathrm{TeV}$, and the data of CDF Collaboration at Tevatron at $\sqrt{S}=1.96 \mathrm{TeV}$.
\end{abstract}

Xth Quark Confinement and the Hadron Spectrum,

October 8-12, 2012

TUM Campus Garching, Munich, Germany

${ }^{*}$ Supported by the Russian Ministry of Science and Education, Contract 14.B37.21.1182

${ }^{\dagger}$ Supported by the RFBR, 12-02-31701-mol-a 
The high-energy regime of Tevatron and LHC, the so called "Regge limit" $\Lambda_{\mathrm{QCD}} \ll \mu \ll \sqrt{S}$, where $\mu$ is a characteristic scale of the relevant hard processes, the contribution of partonic subprocesses involving $t$-channel parton (gluon or quark) exchanges to the production cross section can become dominant. These $t$-channel exchanges obey (quasi-)multi-Regge kinematics ((Q)MRK), when the (groups of) particles produced in the collision are strongly separated in rapidity. For $b$-jet and $b \bar{b}$-dijet inclusive production it means that $b$-jet (MRK) or $b \bar{b}$-dijet (QMRK) is produced in the central region of rapidity, while other particles are produced with large modula of rapidities. The parton Reggeization approach (PRA) [1] is based on the hypothesis of parton Reggeization in $t$-channel exchanges at high energy [2]. Its theoretical background is to be the effective quantum field theory implemented with the non-Abelian gauge-invariant action including fields of Reggeized gluons and Reggeized quarks [2], proposed by L. N. Lipatov in 1995 [3].

We study a $b$-jet production in a region of $b$-quark transverse momenta $p_{T} \gg m_{b}$, where the large logarithms of type $\log \left(p_{T} / m_{b}\right)$ arise to all orders of $\alpha_{s}(\mu)$. They can be resummed in the fragmentation approach, where the main contribution comes from the gluon-to-bottom-pair fragmentation $g \rightarrow b \bar{b}$ which is described by a $b$-quark multiplicity in a gluon-initiated jet $n_{g}(\mu)$.

In the LO of PRA the dominative parton subprocesses for inclusive $b$-jet and $b \bar{b}$-jet production read: $R\left(q_{1}\right)+R\left(q_{2}\right) \rightarrow g(p)(\mathrm{MRK}), R\left(q_{1}\right)+R\left(q_{2}\right) \rightarrow b\left(p_{1}\right)+\bar{b}\left(p_{2}\right)$ (QMRK), which squared amplitudes are presented in the work [4] and $R$ is the Reggeized gluon,

Exploiting the hypothesis of high-energy factorization, the master formula for the inclusive $b$-jet production takes a form, which is also kept for $b \bar{b}$-dijet production [4]:

$$
\frac{d \sigma^{f r a g}(p p \rightarrow b X)}{d p_{T} d y}=\frac{1}{p_{T}^{3}} \int d \phi_{1} \int d t_{1} \Phi_{g}^{p}\left(x_{1}, t_{1}, \mu^{2}\right) \Phi_{g}^{p}\left(x_{2}, t_{2}, \mu^{2}\right) n_{g}(\mu) \overline{|\mathscr{M}(R R \rightarrow g)|^{2}},
$$

where $y$ is the rapidity of $b$-quark, $\phi_{1}$ is the azimuthal angle between $\vec{q}_{1 T}$ and $\vec{p}_{T}, x_{1,2}=\frac{p_{T} \exp ( \pm y)}{\sqrt{S}}$, $t_{2}=t_{1}+p_{T}^{2}-2 \sqrt{t_{1}} p_{T} \cos \left(\phi_{1}\right)$. The unintegrated PDFs $\Phi_{g}^{h}$ of Reggeized gluons in hadrons $h$ are obtained from the integrated one, by the prescription proposed by Kimber, Martin, and Ryskin (KMR) [5], as default, and by the Blümlein approach [6], to estimate the theoretical uncertainty.

We describe the ATLAS data on $b \bar{b}$-jet-production at LHC at $\sqrt{S}=7 \mathrm{TeV}$ [7] well with our LO parton Reggeization approach predictions at the whole presented range of the $b \bar{b}$-dijet invariant mass $M_{j j}$ (Fig. 1, left), the azimuthal angle between the two jets $\Delta \phi$ and the angular variable $\chi$.

For the inclusive $b$-jet transverse-momentum production spectra we account gluon-to-bottompair production mechanism and consider the function of $b \bar{b}$-pair multiplicity $n_{g}(\mu)$ in a gluon jet as a free phenomenological parameter, which we extract from the ATLAS data for the inclusive $b$-jet production spectra [7]. We propose the analytical approximation of $n_{g}(\mu)=A \ln \frac{\mu^{2}}{m_{b}^{2}}$ with $m_{b}=4.75 \mathrm{GeV}$ and $\mu=p_{T} / 4$, and found $A_{K M R}=0.0012$ and $A_{B}=0.0027$, that at the scale $\mu \simeq m_{Z} / 4$ is in agreement with the measurements at the LEP Collider [8]. Using the extracted $n_{g}(\mu)$ we demonstrate good agreement with ATLAS (Fig. 1, right) and CMS data at the CERN LHC, and CDF data at the Fermilab Tevatron [9]. In all cases we find a good agreement between theoretical predictions and experimental data.

A.V.S. is grateful to the Organizing Committee of the Conference "Xth Quark Confinement and the Hadron Spectrum", Munich, 2012, for kind hospitality and financial support. 

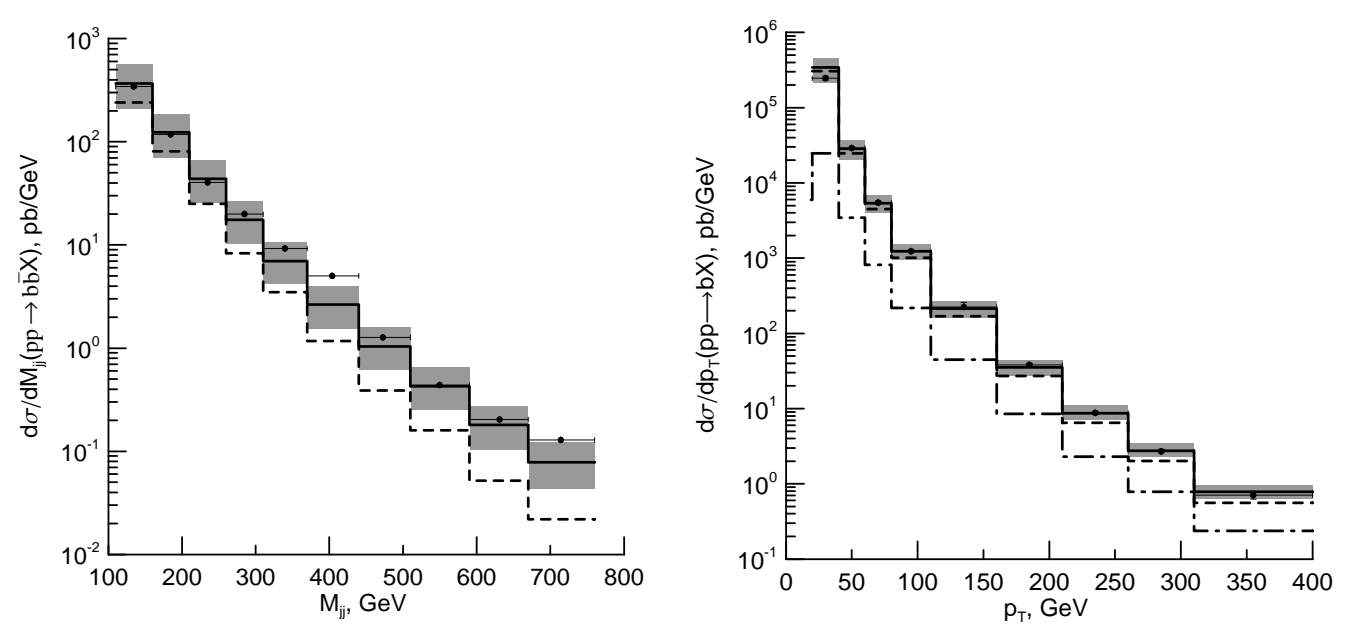

Figure 1: At left: the $b \bar{b}$-dijet cross-section for $b$-jets with $p_{T}>40 \mathrm{GeV},|y|<2.1$, as a function of dijet invariant mass $M_{j j}$. The solid polyline with shaded bands correspond to KMR PDF with theoretical uncertainties, the dashed one - to Blümlein PDF. At right: inclusive differential $b$-jet cross-section as a function of $p_{T}$, the dashed polyline corresponds to the open $b$-quark production, the dashed-dotted one the gluon-to-bottom-pair fragmentation, the solid — sum of all them, $|y|<2.1$. Points - ATLAS data [7].

\section{References}

[1] V. S. Fadin and L. N. Lipatov, Radiative corrections to QCD scattering amplitudes in a multi - Regge kinematics, Nucl. Phys. B406 (1993) 259.

[2] E. A. Kuraev, L. N. Lipatov, and V. S. Fadin, Multi - Reggeon Processes In The Yang-Mills Theory, Sov. Phys. JETP 44 (1976) 443 [Zh. Eksp. Teor. Fiz. 71 (1976) 840]; L. N. Lipatov and M. I. Vyazovsky, Quasi-multi-Regge processes with a quark exchange in the t-channel, Nucl. Phys. B597 (2001) 399 [arXiv: hep-ph/ 0009340$].$

[3] L. N. Lipatov, Gauge invariant effective action for high-energy processes in QCD, Nucl. Phys. $\mathbf{B 4 5 2}$ (1995) 369 [arXiv: hep-ph/9502308].

[4] V. A. Saleev, A. V. Shipilova, Inclusive b-jet and $b \bar{b}$-dijet production at the LHC via Reggeized gluons, Phys. Rev. D 86 (2012) 034032 [arXiv: hep-ph/1201.4640].

[5] G. Watt, A. D. Martin, and M. G. Ryskin, Unintegrated parton distributions and inclusive jet production at HERA, Eur. Phys. J. C 31 (2003) 73 [arXiv: hep-ph/ 0306169 ].

[6] J. Blümlein, On the $k(T)$ dependent gluon density of the proton, Preprint DESY 95-121 (1995) [arXiv: hep-ph/9506403].

[7] ATLAS Collaboration, G. Aadet al., Measurement of the inclusive and dijet cross-sections of b-jets in pp collisions at $\sqrt{s}=7 \mathrm{TeV}$ with the ATLAS detector, Eur. Phys. J. C 71 (2011) 1846 [arXiv: hep-ex/1109.6833].

[8] DELPHI Collaboration, P. Abreu et al., Measurement of the rate of $b$ anti- $b$ b anti- $b$ events in hadronic $Z$ decays and the extraction of the gluon splitting into b anti-b, Phys. Lett. B 462 (1999) 425.

[9] CMS Collaboration, S. Chatrchyan et al., Inclusive b-jet production in pp collisions at $\sqrt{\mathrm{s}}=7 \mathrm{TeV}$, arXiv: hep-ex/1202 .4617; CDF Collaboration, T. Aaltonen et al., Measurement of the inclusive b-jet cross section in p p collisions at $1.96 \mathrm{TeV}$, CDF note 8418 (2006) URL: http://www-cdf.fnal.gov/physics/new/qcd/QCD.html. 